\title{
INFLUENCE OF S AND P DOPING IN A GRAPHENE SHEET
}

\author{
ALE GARCIA $^{1^{*}}$, SE BALTAZAR ${ }^{2}$, AH ROMERO ${ }^{1}$, JF PEREZ ROBLES ${ }^{1}$, A RUBIO ${ }^{3,4}$ \\ ${ }^{1}$ CINVESTAV Unidad Querétaro. Lib. Norponiente \#2000 Fracc. Real de Juriquilla. C.P. 76230. \\ Santiago de Querétaro, México. \\ ${ }^{2}$ Advanced Materials Department, IPICyT. Camino Presa Sn. José 2055, 78216 San Luis Potosí, \\ S.L.P., México. \\ ${ }^{3}$ European Theoretical Spectroscopy Facility (ETSF), Departamento de Fisica de Materiales, , \\ Universidad del Pais Vasco, Edificio Korta, Avd. Tolosa 72, 20018 San Sebastian (Spain). \\ ${ }^{4}$ Unidad de Física de Materiales Centro Mixto CSIC-UPV/EHU and Donostia International \\ Physics Center (DIPC), 20018 San Sebastian (Spain).
}

\begin{abstract}
In this work, we study the electronic and chemical properties of a graphene sheet doped with S or P, by means of ab initio calculations. We consider one, two and three impurity atoms by substitution on the graphene and obtain doping formation energies of 5.78, 7.43 and $10.53 \mathrm{eV}$ for sulphur impurities and 2.73, 0.54 and $1.82 \mathrm{eV}$ for phosphorous impurities. We find that doping induces a large local curvature that tends to increase the system local reactivity. We characterize the electronic structure by the electronic density of states, the electron localization function and the maximally localized Wannier functions. Some potential applications are highlighted.
\end{abstract}

\section{INTRODUCTION.}

Carbon is the sixth element of the periodic table and is listed at the top of group IV. Each carbon atom has six electrons which occupy $1 \mathrm{~s}^{2}, 2 \mathrm{~s}^{2}$, and $2 \mathrm{p}^{2}$ atomic orbitals. The $1 \mathrm{~s}^{2}$ orbitals contain two strongly bound electrons (core electrons), and the $2 \mathrm{~s}^{2} 2 \mathrm{p}^{2}$ orbitals are occupied by 4 weakly bound electrons (valence electrons). The carbon happens to be a very unique element compare with elements in its family. It differs in many ways from Si, Ge, and Sn, other elements of column IV, which all have $\mathrm{sp}^{3}$ bonding in their cubic solid ground states, while carbon in the condensed phase has a hexagonal ground state graphite, where the $\mathrm{sp}^{2}$ bonding and a higher directional anisotropic than the $\mathrm{sp}^{3}$. This is due to the delocalization of the $\mathrm{p}_{z}$ electrons, which for example, promotes the behavior of graphite as an electrical conductor in the in-plane direction. Nevertheless, the out of 
plane conductivity is low due to the Van der Waals interaction between planes [1]. Of all the column IV elements, it is only carbon that can have $\mathrm{sp}, \mathrm{sp}^{2}$ and $\mathrm{sp}^{3}$ bonding, and this is because only carbon has no inner p-electrons [2]. It is a versatile element with different allotropes, including graphite (3D), diamond (3D), the recently discovered nanotubes (1D) and fullerenes (0D)-like structures [3-5]. In particular the carbon nanotubes $(\mathrm{CN})$ are unique nanostructures with remarkable electronic and mechanical properties. They are considered as prototypes for one- dimensional quantum wires. Carbon nanotubes are now being used; for example, in nanometer-sized electronics or strengthen polymer materials, field emission sources [6], Li ion batteries [7], molecular sensors [8-14], gas and hydrogen storage [15-17] and scanning probe tips [18]. However, the lack of solubility and the difficulty in their manipulation, when solved, have imposed great limitations to their use. In order to overcome the lack of chemical reactivity, the modification of carbon nanotubes has been put forward for consideration. A proposed functionalization and purification method for raw $\mathrm{CN}$ material is based on oxidation in acidic media. In this method, most of electronic properties of the functionalized carbon nanotubes remain intact, even though the length of the carbon nanotubes is shortened [19]. This could change the solubility and facilitate the dispersion in a given medium. On the other hand, the doping of carbon nanotubes is another method used to modify the local chemical reactivity. The defect sites increase the reactivity of the nanotube walls, allowing them to be more sensitive to certain chemical species. The chemical reactivity of carbon nanotubes is similar to some other carbon structures, and of graphite in particular [20].

Substitutional doping by $\mathrm{N}$ or B impurities on carbon nanotubes has been a very intense research topic at the experimental and theoretical level during the recent years [21-24]. Initial works have focused on the effect of a single defect on the electronic and transport properties, while further studies have addressed the issue of mesoscopic transport in nanotubes with random distributions of impurities. The influence of defects is of fundamental relevance in the performance of electronic and sensing devices based on carbon nanostructures. Switching from ballistic to either weak or strong localization transport regime is possible above a certain density of defects. Defect sites 
augment the chemical reactivity of nanotubes walls, rendering them sensitive to certain chemical species: “chemical sensors”.

The influence of defects is of fundamental relevance in the performance of electronic and transport properties of carbon materials, in particular for molecular sensor devices. Many researches have doped graphite with B atoms to enhance its crystallinity and graphitization [25, 26]. In ref. [27] is reported that, if $\mathrm{B}$ replaces some $\mathrm{C}$ atoms in a graphene sheet, the electronic structure will contain electronic holes; which are responsible of generating a p-type conductor. Whereas when $\mathrm{N}$ atoms substitute $\mathrm{C}$ atoms, a n-type material is obtained. The incorporation of nitrogen into $s p^{2}$-hybridized carbon materials has been shown to be a promising path to change the mechanical and electronic properties of the material in a controllable manner [28]. For example, aligned $\mathrm{CN}_{x}$ nanotubes could be potentially more efficient in detecting gaseous hazardous species due to the presence of reactive sites on their surface, when compared to pure carbon nanotubes [29]. The introduction of halogen atoms due to their large electronegativity constitutes other possible way of doping single wall $\mathrm{BC}_{3}$ tubules with hole carriers. In fact, halogen doping has shown to lead to superconducting behavior in carbon nanotubes due to the high density of states around the injected hole [30-34].

In this work, we focus in how the electronic and structural properties of a graphene layer are modified by doping with sulphur or phosphorous atoms at different concentration levels. These elements act as donor of electrons and show different changes at the electronic density of states when compared to $\mathrm{N}$ or B doping. Furthermore, it is known, that many electronic (and mechanical) properties of carbon nanotubes can be derived from the corresponding properties of a single graphene sheet by a bandfolding procedure [3], therefore studying the doping of a graphene will provide relevant information not only on the breaking of aromaticity in the two-dimensional honeycomb lattice but also information about the effect of the doping in a carbon nanotube. In general, chemical reactivity is augmented under the presence of doping and vacancies as it was stated in Ref [35]. In the present work, the doping with S or P will provide extra-unbonded valence electrons that could enhance the nanotube's electrical conductivity and chemical reactivity. Other 
potential applications for these structures are absorption of molecules for photovoltaic applications and polymer reinforcement. These could come from the local changes in the electronic structure. The way how this doping could modify the properties of the carbon structures is unknown. To our knowledge, this is the first time, where $\mathrm{P}$ or S are hypothetically introduced in a graphene sheet and the energetic of the substitution sites analyzed.

\section{METHODOLOGY.}

Theoretical analysis of the electronic structure of matter provides a good understanding and a quantitative methodology to describe a great variety of observed phenomena. In particular, we have used Density Functional Theory (DFT) in the Kohn-Sham scheme [36, 37]. Our calculations are based on the implementation of DFT within the CPMD code [38], where orbitals are expanded in plane waves. Only valence electrons are taken into account by using normalized pseudopotentials, generated in the Trouiller-Martins scheme [39]. The exchange-correlation energy was approximated by using BLYP [40, 41]. We used periodic boundary conditions along the graphene plane and a large empty box along the c-axis, typically of $8 \AA$. We did check the convergence of our results with respect to the box size perpendicular to the sheet. The energy cutoff value used was 60 Ry, which has proven to be quite accurate to describe carbon systems [42]. Specific checks were performed to see the dependence of our results as function on the cutoff. Forces were converged up to $0.01 \mathrm{eV} / \AA$.

The minimal energy structures were obtained by using the LBFGS algorithm [43]. The in-plane (a) and out-of-plane (b) cell parameters were optimized for the pristine graphene as well as for the doped sheets. We used a graphene sheet with 72 atoms (carbons and impurities). In particular, for the case of the pure graphene, we obtain $a=b=14.826 \AA$, which gives a cell parameter of $2.471 \AA$, close to the experimental value of $2.46 \AA$ [44]. The calculated C-C bond length is $1.42 \AA$, also close to the experimental value of $1.421[45,46]$.

To obtain the lowest energy configuration, we considered different substitutional positions for the doping atoms. We label the considered impurities configurations as shown in figure 1 . We have 
introduced the following notation; considering $\mathrm{A}$ as the impurity reference; the combinations for two atoms are $\mathrm{AB}, \mathrm{AC}$ and $\mathrm{AD}$. For three atoms the combinations are $\mathrm{ABC}, \mathrm{ABD}, \mathrm{ABE}$ and $\mathrm{BGF}$. We did also consider a pyridinic site which corresponds to the sites BGF without the center carbon (A).

Maximally Localized Wannier functions were obtained by following the description in Ref. [47]. These are obtained through a unitary transformation of the Kohn-Sham orbitals with the constraint of minimal wave function spread ( $\min <\mathbf{r}^{2}>$ ). The location of the Wannier function centers (WFCs) provides a means of reliably determining the existence of a bond and the shape type will describe the bonding type. In addition, defect states such as lone pairs of electrons can be also identified. The Electron Localization Function (ELF) was calculated as defined in [48], and the Electron Density of States was obtained through the projection of the dispersion relation energies with a 7x7x1 Monkhorst-Pack grid [49] and 0.15 eV broadening of every energy level.

The formation energy was calculated as follows:

$E_{\mathrm{F}}=E_{\mathrm{T}}+n \mu_{\mathrm{C}}-\mathrm{E}_{\mathrm{g}}-\mathrm{m} \mu_{\mathrm{i}} \quad$,

where $\mathrm{E}_{\mathrm{T}}$ refers to the total energy of a graphene sheet after doping, $\mathrm{n}$ denote the number of $\mathrm{C}$ atoms which are substituted and $m$ the included impurity atoms. $E_{\mathrm{g}}$ is the total energy of the pure carbon graphene sheet, $\mu_{\mathrm{C}}$ and $\mu_{\mathrm{i}}$ are the chemical potentials of the carbon and impurity atoms respectively. The chemical potential for carbon was taken from a graphene sheet calculated as $E_{g} / N_{C}$ as defined in [50]; where $\mathrm{N}_{C}$ is the number of carbons in the supercell $\left(\mathrm{N}_{\mathrm{C}}=72\right.$ in our case). The doping chemical potential was calculated from the atomic energies from dimers $\left(\mu_{\mathrm{i}}=\mathrm{E}_{\text {dimer }} / 2\right.$, $\mathrm{i}$ being $\mathrm{S}$ or P), because those two species are found as dimers in the gas phase.

\section{RESULTS AND DISCUSSION.}

We first consider the substitutional doping of a single atom, as it is reported in table IV, the lowest energy of formation corresponds to the phosphorous case. 
As a next step, we look at the doping of two atoms. Both atoms are located as close as possible within the carbon-network. The lowest doping energy configuration for the two impurity atoms is the $\mathrm{AD}$ combination, as shown in table I and figure 2. Figure 2 also shows how the graphene network is locally deformed when the doping atoms are introduced. In the case of phosphorous, both atoms tend to be on the same side and are displaced $2.03 \AA$ with respect to the sheet plane, whereas, when the network is doped with sulphur atoms, the two atoms distribute themselves on top and below the graphene sheet, at a distance of $0.93 \AA$. Figure 3 shows the bond distances after doping. In order to check our procedure, we did compare the distance C-P with organic molecules such as phosphine, methyl-phosphine and phosphinidenes (of the order of $1.86 \AA$ ) [51, 52]. The obtained bond lengths are very close to the reported bond values, even though in somewhat different systems. The bond C-S was compared with dimethyl disulfide and ethyl methyl sulfide $[52,53]$, where the bond length for these organic molecules is $1.82 \AA$ in average. Bond distances are also close to our calculation, as seen in figure 3. The Maximally Localized Wannier Functions around the doping atoms are shown in Figures 4 and 5. It is clear that there is a covalent bond between the impurity and the carbon atoms as well as lone pairs on the impurities atoms. The increase in local reactivity clearly comes from the presence of these lone pairs. Table II shows the Maximally Localized Wannier Function Spreads (WFS) for the states around the doping atoms, as defined in reference [36]. Comparing the lone pairs WFS for sulphur and phosphorous, we see that phosphorous has a slightly larger value, indicating a larger availability of these electrons. If we add the fact that both $\mathrm{P}$ doping atoms are located on the same side with respect to the graphene sheet, and that the lone pair Wannier Functions is more isotropic (compare figure 5b with $4 \mathrm{~b}$, with the same isovalue), we conclude that $\mathrm{P}$ doping has a larger reactivity when compared to sulphur.

In the case of three atom doping (without creating a vacancy), the BGF configuration happens to be the most stable one (see table III). Figure 6 shows the lowest energy structures for the two impurity cases, S and P. A larger local deformation is observed when compared to the 2 atoms case. For the sulphur atoms, they stand at a distance of $1.61 \AA$ from the plane and all atoms are on the same side. 
In the case of phosphorous the atoms stand at $2.07 \AA$ from the plane, similar to the 2 atom doping. The $\mathrm{C}$ atom centered in between the impurities is displaced $1.99 \AA$ respect to graphene sheet in the phosphorous case and $0.81 \AA$ in the sulphur case. We also find that in particular for $\mathrm{P}$ and $\mathrm{S}$, the pyridinic site is the one with the lowest formation energy for 3 atoms doping. The minimal geometries are reported in figures 11 and 12.

Table IV summarizes the formation energies for all doping cases we have considered. As shown, $\mathrm{P}$ would be always preferred over S. Our calculations also show that there is not a large difference between 3 close doping atoms and a pyridinic site, indicating that both possibilities could be obtained experimentally.

With respect to the electronic properties, for 3 atoms doping, the lone pair Wannier function on top of the doping atom has a larger spread in $\mathrm{P}$ than $\mathrm{S}$ (see table $\mathrm{V}$, figure $7 \mathrm{~b}$ and $8 \mathrm{~b}$ ). This indicates that there is a facility of the P lone pair electrons to get involved in a nucleophilic attack. Figures 7a and 8a show the covalent bond between the impurities and carbon atoms. By looking at the LUMO (Lowest Unoccupied Molecular Orbital) state (see figure 9), we see that there is a localization of the electronic density along the C-C bond, for the S atoms, and on top of the C atoms, for the $\mathrm{P}$ atoms. In either case, for the three atoms doping, the LUMO density is localized more on the neighbour carbon atoms. However, in the three sulphur atoms case, the density concentration on the atoms is larger than in the phosphorous case. In the HOMO (Higher Occupied Molecular Orbital), the electronic density is more localized on the doping atoms, indicating that under an electrophilic attack, the doping atoms are going to be more available for reaction than any other part in the system. The two previous observations are corroborated by the ELF (Electronic Localization Function) (see figure 10). The ELF is a tool to describe chemical bonding and provides an alternative to look into the chemical bonding. Figure 10 shows the charge distribution and the reactivity increase around the doping atoms.

To unravel the influence of the spin, we have performed calculations by using the local spin density approximation. We obtain a difference in total energy of 0.04 and $0.15 \mathrm{eV}$ for sulphur and 
phosphorous respectively with respect to a non-local spin density calculation. We conclude that even though, there could be a spin localization on the system, it is not very large and would not change the main results of the present work.

Coming back to the doping configurations, a different type of doping can be obtained if a pyridinic site is taken in to account. As discussed in ref. [29, 54-56] this could happen in carbon nanotubes when they are doped with B or N, where a much larger reactivity is observed. In those works, it is confirmed that $\mathrm{N}$ is responsible for introducing donor states in pyridinic sites close the Fermi Level. This result is in contrast to the $\mathrm{B}$ case $[56,57]$. Figures $11 \mathrm{a}$ and $12 \mathrm{a}$ show the minimal structure when the graphene sheet is doped with a pyridinic site with S and P respectively. For the case of S, the impurity atoms remain in the graphene plane, whereas for $\mathrm{P}$ there is a small tilt with atoms leaving the plane almost up $1.52 \AA$. When we compare both types of pyridinic sites by using the Wannier Functions, the corresponding lone pair states are more extended in the P doping than with S. The WFS are reported in table VI.

In order to characterize the potential use in conduction or molecular absorption, the electronic properties were characterized by looking at the electronic density states. Figure 13 and 14 exhibits the spectra for doped a graphene sheet when the sheet is doped with two and three atoms (only the lowest energy configurations). The basic shape of the e-DOS remains almost the same with respect to the pristine sheet, with the appearance of a peak above the Fermi level which depends on the type of doping. Interestingly enough, the peak intensity depends on the localization on the impurity atoms. In particular, the highest intensity is obtained for the pyridinic case around $0.47 \mathrm{eV}$. A similar result is obtained for the case of $\mathrm{N}$-doping but with energy of $0.18 \mathrm{eV}$ [54]. We should also point out that in previous theoretical investigations, when $\mathrm{N}$ is placed substitutionally and fully coordinated to the underlying carbon lattice in a single walled nanotube, the system shows a quasibond electronic of states approximately $0.53 \mathrm{eV}$ from the Fermi Level [58]. For P doping with 2 atoms, the peak occurs around $0.62 \mathrm{eV}$, while doping with 3 atoms is observed at $0.37 \mathrm{eV}$. When the defects density increases, the peaks position in the electronic density of states not only change 
(getting close to the Fermi level) but also they could be randomly distributed on the network, increasing in electronic localization [59]. Another effect is related to the electronic delocalization due to the introduction of defects. For example, in the case of a pyridinic site with sulphur doping, one electron is completely delocalized on the graphene sheet, as shown from the Maximally Localized Wannier Functions of that particular state (see figure 16). This was not observed in the case of P doping, even though, there is a state which become spreader around the phosphorous atom (see figure 15). In the sulphur case, the dispersion of this electronic state is larger due to the delocalization and the contribution to the density of states is smaller.

\section{CONCLUSIONS.}

In this paper, we demonstrate, by theoretical means, that doping of a graphene sheet with $\mathrm{S}$ or $\mathrm{P}$ is possible with an average binding energy of $0.19 \mathrm{eV}$ and $0.30 \mathrm{eV}$ for phosphorous and sulphur respectively. The minimal energy configurations were obtained and a structural characterization was discussed. In our results, we observed that the lowest stable configuration is for P doping, independent of the number of doping atoms. Maximally Localized Wannier Functions describe rather nicely the covalent bonds along the C-doping atoms, as well as then lone pairs on the impurity atoms. A large spread of the lone pair Wannier states was observed for the P doping when compared with the S doping. The LUMO state is more distributed around the. An analysis on the electronic density of states indicates the appearance of a state above the Fermi Level coming from the doping atoms.

Between the different doping levels that we have considered, we did include also the possibility of having pyridinic sites. We find that this possibility is close in doping formation energy to the doping with three atoms in the unit cell. No large changes in the electronic density of states are observed when compared with the 3 atoms doping without a vacancy. In the case of sulphur we find that one of the maximally localized Wannier states is spread almost along the whole sheet, this 
will have implications on the conduction properties of the system. We conclude, based on the electronic analysis we have performed, that P doping could be quite reactive and will potentially improves the chemical properties of carbon nanostructures when compare with S doping. Finally our observations feed the possibility of using these doped networks in nanocatalysis and sensor nanostructures, either in graphene or in carbon nanotubes1. In summary, more striking advances in both modelling and experiments are ready to come in the near future: we have to be ready to discover some "surprises" to stem from the new physical and chemical properties of this whole class of nanocomposite materials.

\section{Acknowledgments}

The Mexican group thanks the support of Conacyt with Grant F- 59853-J. We also acknowledge the computer allocation time on the CNS, Ipicyt, Mexico. AR acknowledge support by the European Community Network of Excellence Nanoquanta (NMP4-CT-2004-500198), SANES (NMP4-CT2006-017310), DNA-NANODEVICES (IST-2006-029192), NANO-ERA-Chemistry projects, Spanish MEC (FIS2007-65702-C02-01), Basque Country University (SGIker ARINA). We thankfully acknowledge the computer resources, technical expertise and assistance provided by the Barcelona Supercomputing Center - Centro Nacional de Supercomputación.

\section{REFERENCES.}

[1] D. D. L. Chung. J. Mater. Sci. 39, 2645-2661, (2004).

[2] T. W. Ebbesen. Carbon nanotubes. Preparation and Properties. Press. 1998.

[3] M.S. Dresselhaus, G. Dresselhaus and P.C. Eklund, Science of fullerenes and Carbon Nanotubes (Academic Press, New York 1996).

[4] M. Terrones , Annu. Rev. Mater. Res. 33, 419-501 (2003). 
[5] M. Terrones , H. Terrones. Phil. Trans. R. Soc. London A 361, 2789, (2003).

[6] J.C. Charlier, M. Terrones, M. Baxendale, V. Meunier, T. Zacharia. Nanolett. 2, 1191-1195, (2002).

[7] E. Frackowiak, F. Beguin. Carbon 40, 1775-1787, (2002).

[8] J. Kong, N.R. Franklin, C.W. Zhou, M.G. Chapline, S. Peng. Science 287, 622-625, (2000).

[9] P.G. Collins, K. Bradley, M. Ishigami, A. Zettl. Science 287, 1801-1804, (2000).

[10] S.S. Wong, E. Joselevich, A.T. Woolley, C.L. Cheung, C.M. Lieber. Nature. 394, 52-55, (1998).

[11] O.K. Varghese, P.D. Kichambre, D. Gong, K.G. Ong, E.C. Dickey, C.A. Grimes. Sensors Actuators B. 81, 32-41, (2001).

[12] S. Chopra, A. Pham, J. Gaillard, A. Parker, A.M. Rao. Appl. Phys. Lett. 80, 4632-4634, (2002).

[13] J.R. Wood, H.D. Wagner. Appl. Phys. Lett. 76, 2883-85, (2000).

[14] J. R. Wood, Q. Zhao, M. D. Frogley, E. R. Meurs, A. D. Prins. Phys. Rev. B. 62, 7571-7575, (2000).

[15] A. Chambers, C. Park, R.T.K. Baker, N.M. Rodriguez. J. Phys. Chem. B. 102, 4253-4256, (1998).

[16] P. Chen, X. Wu, J. Lin, K. L. Tan. Science 285, 91-93, (1999).

[17] M. Hirscher, M. Becher, M. Haluska, A. Quintel, V. Skakalova. J. Alloys Comp. 330, 654-658 (2002).

[18] H.J. Dai, J.H. Hafner, A.G. Rinzler, D.T. Colbert, R.E. Smalley. Nature 384, 147-50, (1996).

[19] D. Tasis, N. Tagmatarchis, V. Georgakilas, M. Prato. J. Chem. Eur. 9, 4000-4008, (2003).

[20] D. Tasis, N. Tagmatarchis, A. Bianco, M. Prato. Chem. Rev. 106, 11015-11036, (2006).

[21] H.J. Choi, J. Ihm, S.G. Louie and M.L. Cohen, Phys. Rev. Lett. 84, 2917, (2000).

[22] C.C. Kaun, Phys. Rev. B. 65, 205416, (2002).

[23] A.H. Nevidomskyy, G. Csanyi, M.C. Payne, Phys. Rev. Lett. 91, 105502 (2003). 
[24] H-F. Song, J-L. Zhu, J-J. Xiong, Phys. Rev. B. 66, 245421 (2002).

[25] C.E. Lowell, J. Am. Ceram. Soc. 50, 142, (1966).

[26] C.T. Hach, Carbon. 37, 221, (1999).

[27] M. Terrones, A. Jorio, M. Endo, A.M. Rao, Y.A. Kim, T. Hayashi, H. Terrones, J.-C. Charlier, G. Dresselhaus and M.S. Dresselhaus. Materials Today. 7, 10, 30-45, (2004) .

[28] Yuchen Ma, A. S. Foster, A. V. Krasheninnikov and R. M. Nieminen. Phys. Rev. B. 72, 205416, (2005).

[29] F. Villalpando-Paez, A.H. Romero, E. Munoz-Sandoval, L.M. Marinez, H. Terrones and M. Terrones Chem. Phys. Lett. 386, 1-3, 137-143, (2004).

[30] Y. Miyamoto, A. Rubio, M.L. Cohen and S.G. Louis. Phys. Rev. (Rapid Communications) B. 50, 4976-4979 (1994).

[31] Y. Miyamoto, A. Rubio, M.L. Cohen and S.G. Louis. Phys. Rev. B. 50, 18360-18366 (1994).

[32] Y. Miyamoto, A. Rubio, X. Blase, M.L. Cohen and S.G. Louis. Phys. Rev. Lett. 74, 2993-2996 (1995).

[33] A. Rubio, Y. Miyamoto, X. Blase, M.L. Cohen and S.G. Louis. Phys. Rev. B. 53, 4023-4026 (1996).

[34] G. G. Fuentes, E. Borowiak-Palen, M. Knupfer, T. Pichler, J. Fink, L. Wirtz, and A. Rubio. Phys. Rev. B. 69, 245403-1,6 (2004).

[35] C. Gómez-Navarro, P.J. De Pablo, J. Gómez-Herrero, B. Biel, F.-J. Garcia-Vidal, A. Rubio and F. Flores, Nature Mater. 4, 534 (2005).

[36] W. Khon and L. J. Sham. Phys. Rev. 140, 4A, (1965).

[37]P. Hohenberg and W. Khon. Phys. Rev. 136, 3B, (1964).

[38] CPMD, Copyright IBM Corp 1990-2006, Copyright MPI für Festkörperforschung Stuttgart (1997-2001).

[39] N.Troullier and J.L. Martins. Phys. Rev. B. 43, 3, (1991).

[40] A.D. Becke. Phys. Rev. A. 38, 6., 3098-3100, (1988). 
[41] C. Lee, W. Yang, and R.G. Parr. Phys. Rev. B. 37, 2, 785-789, (1988-I).

[42] A. H. Romero, D. Sebastiani, R. Ramírez and M. Kiwi. Chem. Phys. Lett. 366, 134-140, (2002).

[43] S. R. Billeter, A. Curioni and W. Andreoni, Comput. Mat. Sci. 27, 437-445 (2003).

[44] M.S. Dresselhaus, G. Dresselhaus, R. Saito, A. Jorio. Phys. Rep. 409, 47-99 (2005).

[45] H. Yanagisawa, T. Tanaka, Y. Ishida, M. Matsue, E. Rokuta, S. Otani and C. Oshima. Phys. Rev. Lett. 93, 17 (2004).

[46] W.A. Harrison, Electronic Structure and the Properties of Solids: The Physics of the Chemical Bond. San Francisco: Freeman, 1980:90.

[47] G. Berghold, C.J. Mundy, A.H. Romero, J. Hutter, and M. Parrinello Phys. Rev. B. 61, 10040-10048 (2000).

[48] A.D. Becke, K.E. Edgecombe. J. Chem. Phys. 92, 5397-5403, (1990).

[49] H.J. Monkhorst and J.D. Pack. Phys. Rev. B 13, 5188 - 5192 (1976)

[50] M. Rossi, A. Fazzio and A.J.R. da Silva. Theoretical study of N-complexes in carbon nanotubes. Cond-Mat 0701602 (2007).

[51] S. Grigoleit, A. Alijah, A.B. Rozhenko, R. Streubel, W.W. Schoeller. J. Organomet. Chem. 643-644, 223-230 (2002).

[52] CRC Handbook of Chemistry and Physics, 80th edition (June 1999).

[53] M. Wierzejewska, M. Saldyka. J. Mol. Struct. 786, 33-38 (2006).

[54] M. Terrones, P.M. Ajayan, F. Banhart, X. Blase, D.L. Carroll, J-C. Charlier, R. Czerw, B. Foley, N. Grobert, R. Kamalakaran, P. Kohler-Redlich, M. Ruhle, T. Seeger and H. Terrones. Appl. Phys. A. 74, 355-361 (2002).

[55] R. Czerw, M. Terrones, J-C. Charlier, X. Blase, B. Foley, R. Kamalakaran, N. Grobert, H. Terrones, D. Tekleab, P. M. Ajayan, W. Blau, M. Ruhle and D. L. Carroll. Nanolett. 1, 9, 457-460 (2001).

[56] X. Blase, J-C. Charlier, A. De Vita and R. Car. Phys. Rev. Lett. 83, 24 (1999). 
[57] D.L Carroll, Ph. Redlich, X. Blase, J-C, Charlier, S. Curran, P.M. Ajayan, S. Roth and M. Ruhle. Phys. Rev. Lett. 81, 2332-2335 (1998).

[58] H.J. Choi, J. Ihm, S.G. Louie and M.L. Cohen. Phys. Rev. Lett. 84, 2917 - 2920 (2000)

[59] J-C. Charlier, X. Blase and S. Roche. Rev.Mod.Phys. 79, (2007).

\section{FIGURES CAPTIONS.}

Figure 1. Doping sites as considered on the graphene sheet. Different combinations of doping involve the sites label in this figure.

Figure 2. Calculation of the minimal energy structure on a graphene sheet doped with two atoms (a) of $S(b)$ or $P(c)$. To doped graphene sheet with $S$ both atoms are displacement $0.93 \AA$ respect to graphene plane. If the structure is doped with $\mathrm{P}$ the atoms are displaced $2.03 \AA$, these atoms tend to be on the same side.

Figure 3. Shown the distances obtained in our calculations between C-C and C-S bond and C-P respectively. These results are very close to experiment values.

Figure 4. Maximally Localized Wannier Functions for a 2 atoms S-doped graphene sheet a) along the C-S bond, b) on the lone pair. The main observation is the covalent bond formation between the carbon and sulphur atoms. Blue color represents the negative value for the isosurface and green colour the positive value.

Figure 5. Maximally Localized Wannier Functions for a 2 atoms P-doped graphene sheet 
a) along the C-P bond, b) on the lone pair. The main observation is the covalent bond formation between the carbon and phosphorous atoms. Blue color represents the negative value for the isosurface and green colour the positive value.

Figure 6. Calculation of the minimal energy structure of a graphene sheet doped with three atoms. b) Sulphur, c) Phosphorous. In both cases the main observation is a local deformation. In the sulphur case the atoms stand at a distance of $1.61 \AA$ from the plane. In the case of phosphorous the atoms stand it $2.07 \AA$, in both cases the impurity atoms are on the same side.

Figure 7. Calculation of the Maximally Wannier Functions for a graphene sheet with three Sulphur atoms a) along the covalent bond C-S, b) Lone pair for the S atom and c,d) Lone pair for the $\mathrm{C}$ atom of the $\mathrm{B}$ position.

Figure 8. Maximally Wannier Functions for a graphene sheet with three Phosphorous atoms a) along the covalent bond $\mathrm{C}-\mathrm{P}, \mathrm{b}$ ) Lone pair for the $\mathrm{C}$ of the $\mathrm{B}$ position in a graphene sheet with three phosphorous atoms.

Figure 9. Lowest Unoccupied Molecular Orbital for doped graphene sheet a) for two sulphur atoms, b)for two phosphorous atoms, c)for three sulphur atoms, d)for three phosphorous atoms. The isovalue used for LUMO was of 0.0005 . 
Figure 10. Electronic Localization Function for a) three sulphur and b) phosphorous atoms.

The isovalue used for ELF was 0.67 .

Figure 11. Minimal energy structure and Maximally Wannier Functions to a graphene sheet with three sulphur atoms without the carbon in the center (A). a) Pyridinic structure b) lone pairs for the one Sulphur atom.

Figure 12. Calculation of the minimal energy structure and Maximally Wannier Functions to a graphene sheet with three Phosphorous atoms without the carbon in the center (A). a, b) Pyridinic structure c, d) lone pairs for the one Phosphorous atom.

Figure 13. Electronic Density of States of a pristine graphene compare with $\mathrm{P}$ doped. Red line is refer to a graphene sheet doped with two phosphorous atoms, the blue line to a graphene sheet doped with 3 phosphorous atoms and green line to a phosphorous pyridinic site.

Figure 14. Electronic Density of States of a pristine graphene compare with S doped. Red line is refer to a graphene sheet doped with two sulphur atoms, the blue line to a graphene sheet doped with 3 sulphur atoms and green line to a sulphur pyridinic site.

Figure 15. Maximally Localized Wannier Function for the pyridinic state with the maximum spread (2.51 Å) for P-doped graphene sheet. 
Figure 16. Maximally Localized Wannier Function for the pyridinic state with the maximum

spread (3.78 ̊̊) for S-doped graphene sheet.

\section{TABLE CAPTIONS}

Table I. Formation Energy to possible configurations for 2 atoms.

Table II. Maximally Localized Wannier Function Spread value in the lone pairs and covalent bond $(\mathrm{X}-\mathrm{C})$ for 2 atoms. $\mathrm{X}$ is the impurity ( $\mathrm{P}$ or $\mathrm{S})$.

Table III. Formation Energy to possible configurations for 3 atoms.

Table IV. Energy Formation for Sulphur and Phosphorous.

Table V. Spread value in lone pairs for $C_{B}$ and impurity atoms and covalent bond $\left(A-C_{B}\right)$

for 3 atoms . A is the impurity ( $P$ or $S$ ).

Table VI. Spread value in lone pairs for $\mathrm{C}$ and impurity atoms and covalent bond (B-C) for

pyridinic case. $B$ is the impurity ( $P$ or $S$ ). 


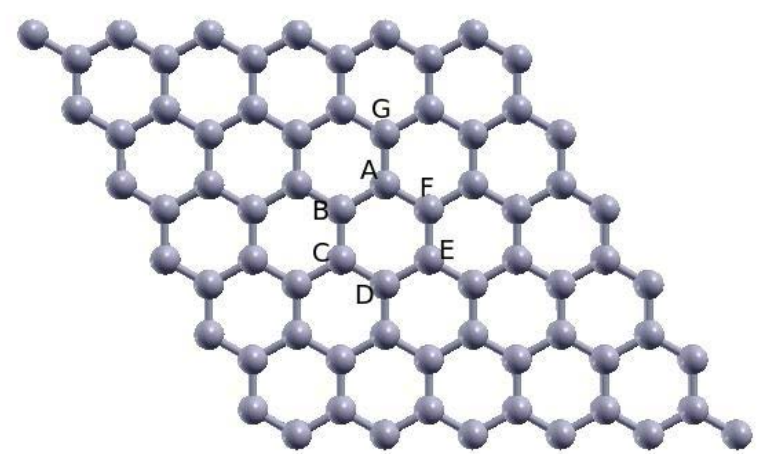

FIGURE 1.

AUTHOR: ALE GARCIA.

ARTICLE: INFLUENCE OF S AND P IN A GRAPHENE SHEET 


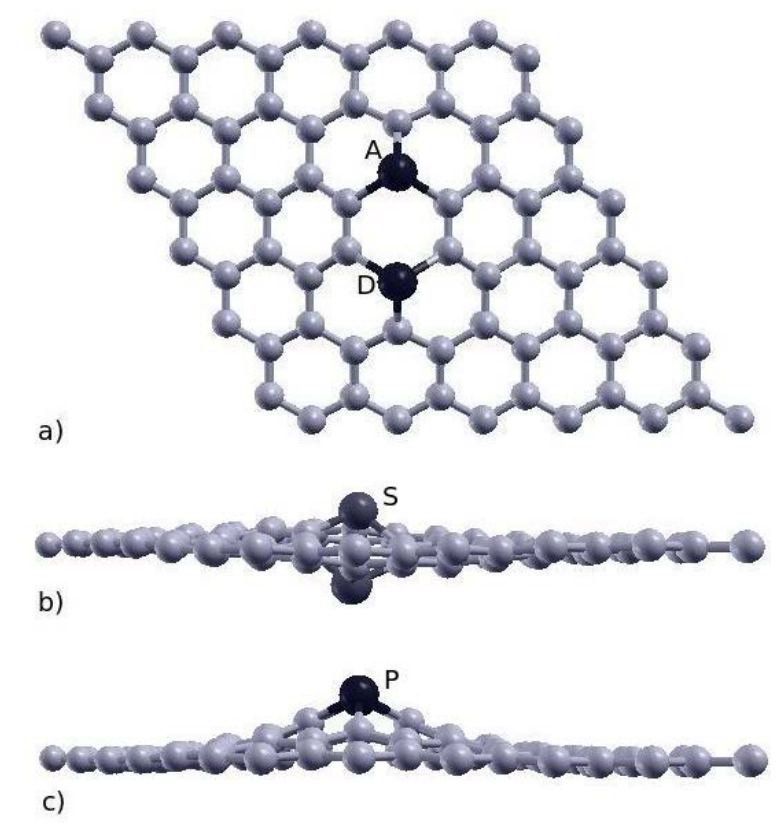

FIGURE 2.

AUTHOR: ALE GARCIA.

ARTICLE: INFLUENCE OF S AND P IN A GRAPHENE SHEET 


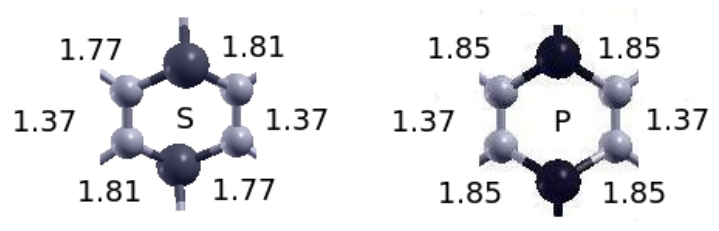

FIGURE 3.

AUTHOR: ALE GARCIA.

ARTICLE: INFLUENCE OF S AND P IN A GRAPHENE SHEET 


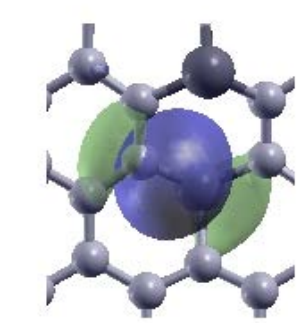

a)

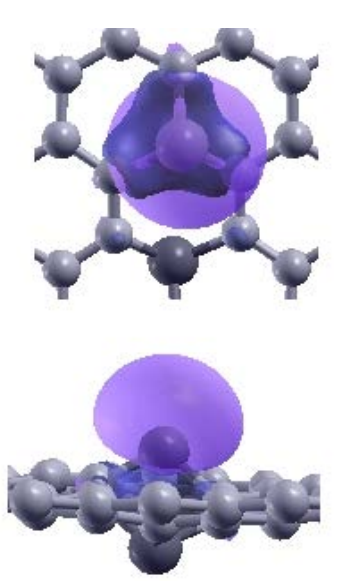

b)

FIGURE 4.

AUTHOR: ALE GARCIA.

ARTICLE: INFLUENCE OF S AND P IN A GRAPHENE SHEET 


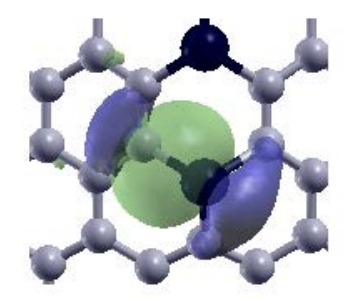

a)

b)

FIGURE 5.

AUTHOR: ALE GARCIA.

ARTICLE: INFLUENCE OF S AND P IN A GRAPHENE SHEET 


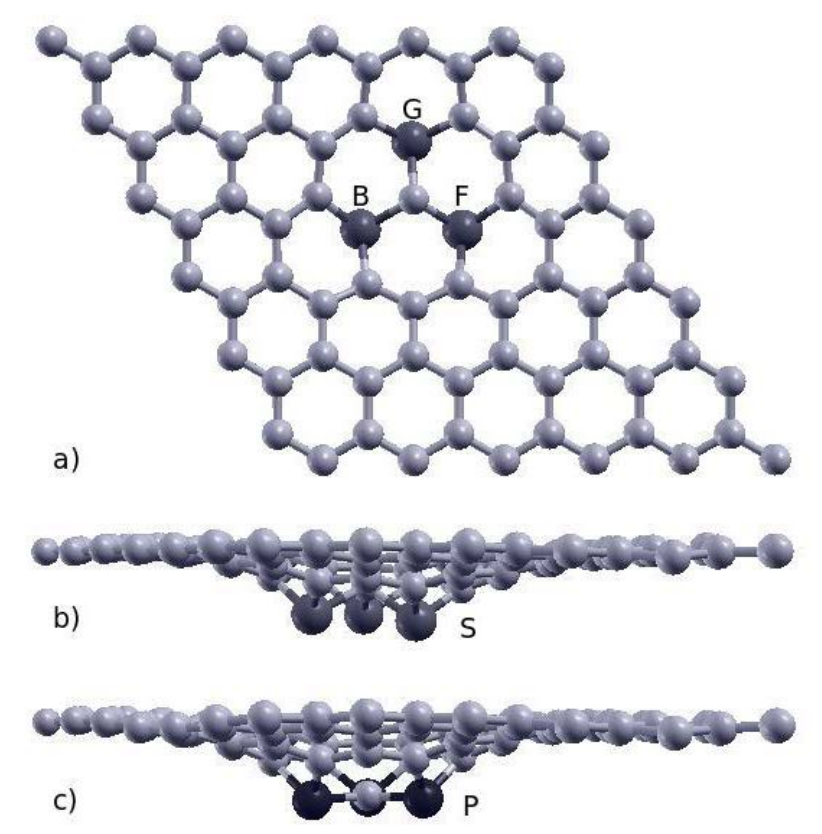

FIGURE 6.

AUTHOR: ALE GARCIA.

ARTICLE: INFLUENCE OF S AND P IN A GRAPHENE SHEET 


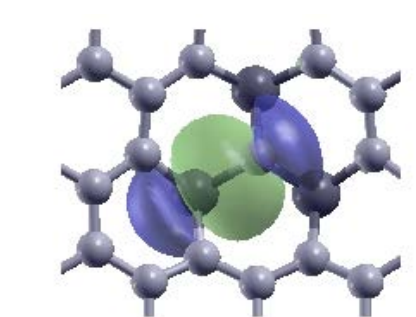

a)

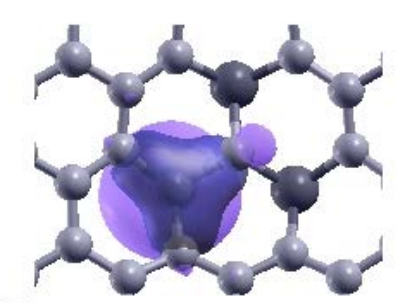

b)

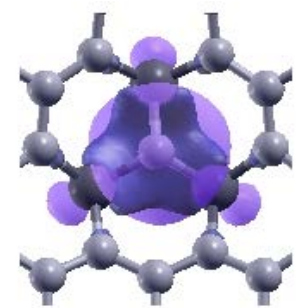

c)

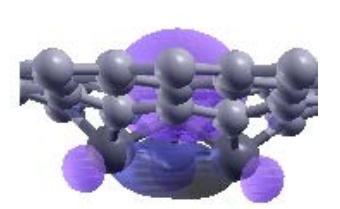

d)

FIGURE 7.

AUTHOR: ALE GARCIA.

ARTICLE: INFLUENCE OF S AND P IN A GRAPHENE SHEET 


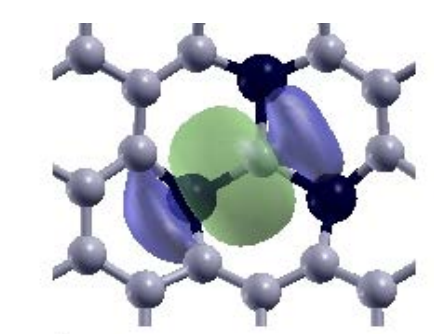

a)

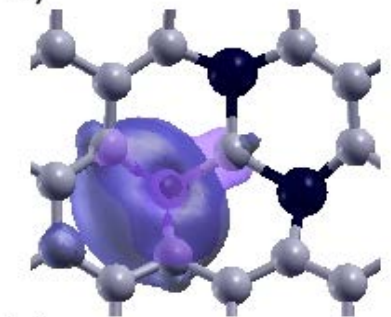

b)

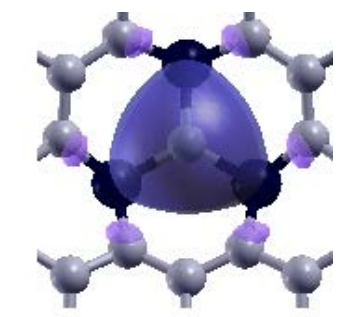

c)

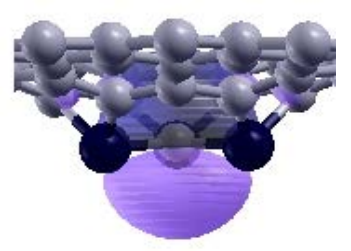

d)

FIGURE 8.

AUTHOR: ALE GARCIA.

ARTICLE: INFLUENCE OF S AND P IN A GRAPHENE SHEET 

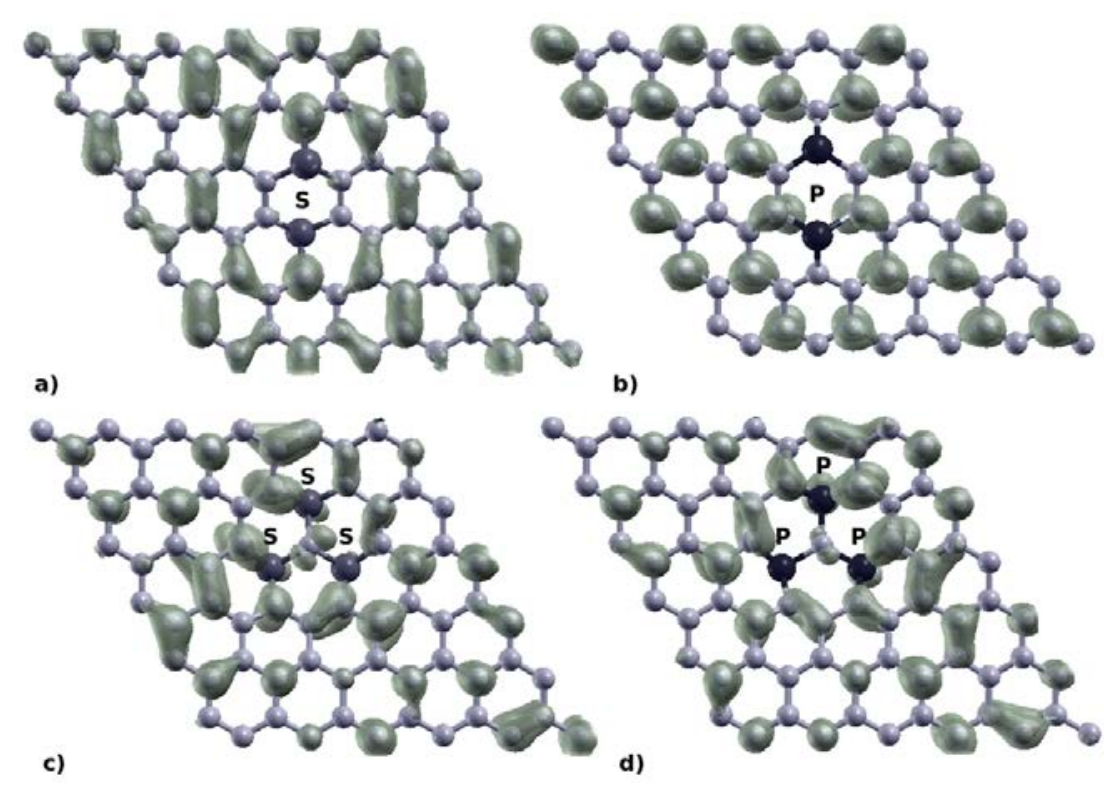

FIGURE 9.

AUTHOR: ALE GARCIA.

ARTICLE: INFLUENCE OF S AND P IN A GRAPHENE SHEET 


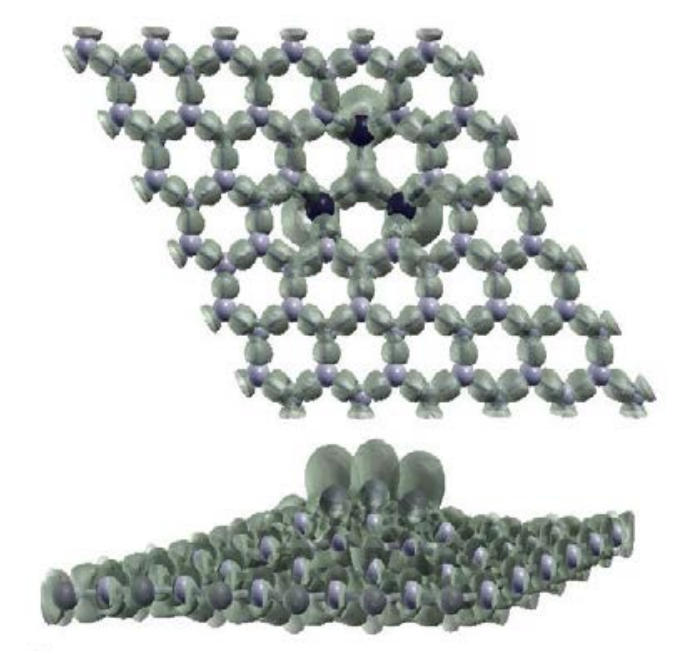

a)

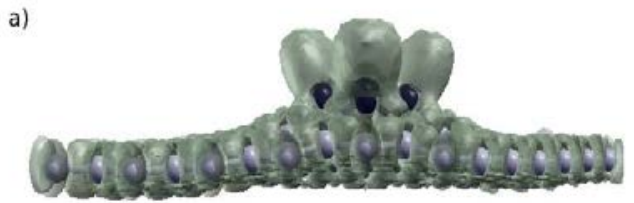

b)

FIGURE 10.

AUTHOR: ALE GARCIA.

ARTICLE: INFLUENCE OF S AND P IN A GRAPHENE SHEET 


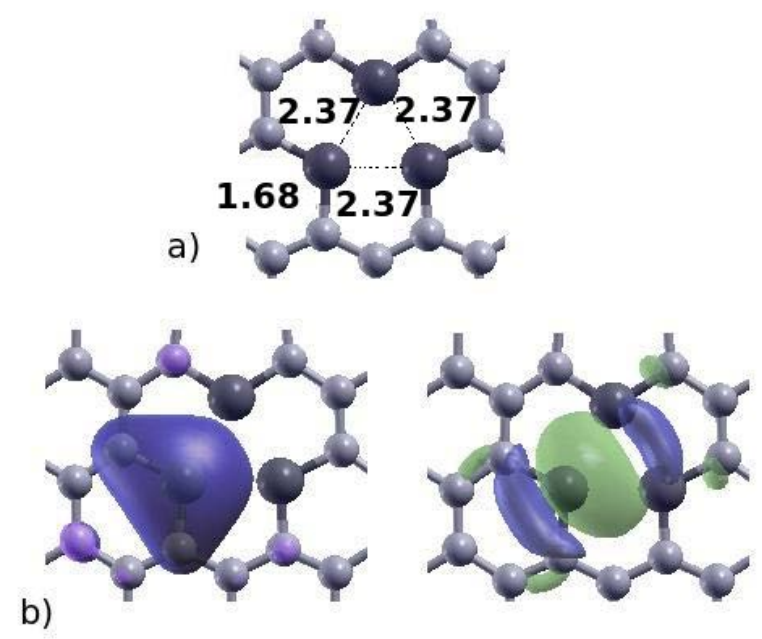

FIGURE 11.

AUTHOR: ALE GARCIA.

ARTICLE: INFLUENCE OF S AND P IN A GRAPHENE SHEET 


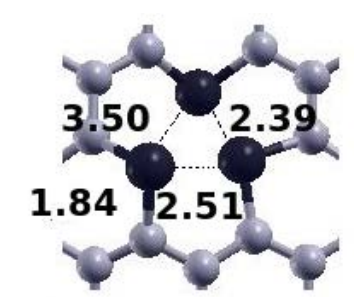

a)

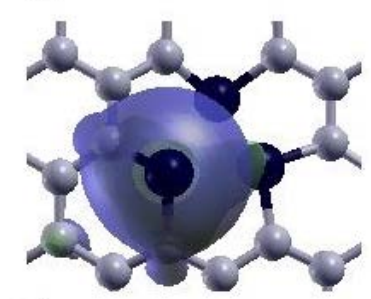

c)

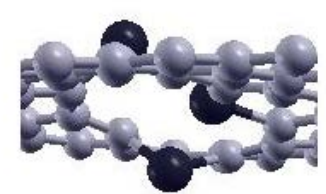

b)

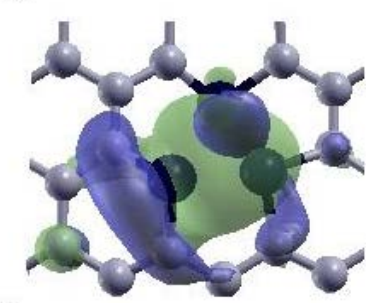

d)

FIGURE 12.

AUTHOR: ALE GARCIA.

ARTICLE: INFLUENCE OF S AND P IN A GRAPHENE SHEET 


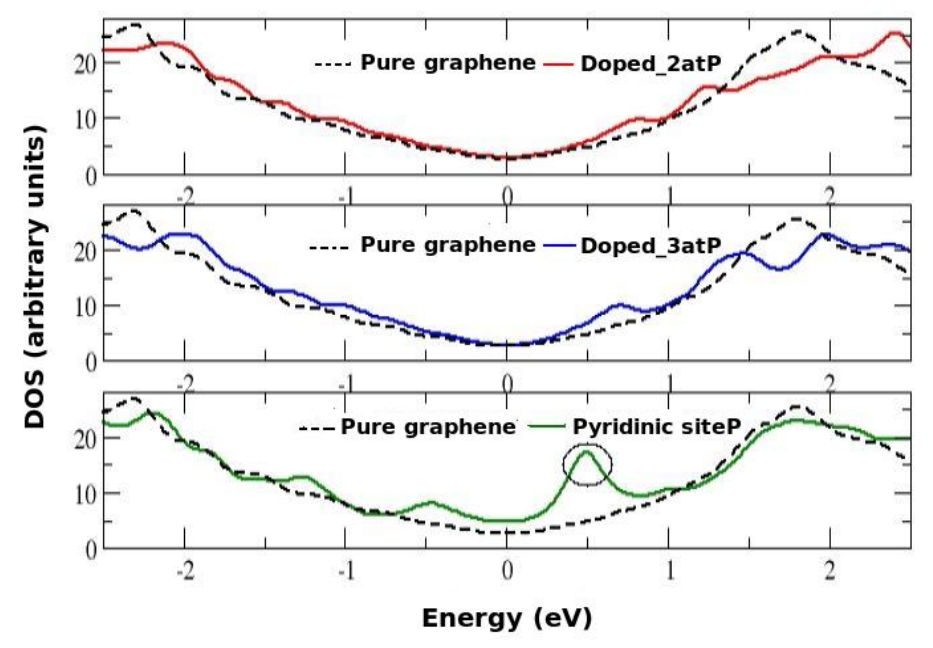

FIGURE 13.

AUTHOR: ALE GARCIA.

ARTICLE: INFLUENCE OF S AND P IN A GRAPHENE SHEET 


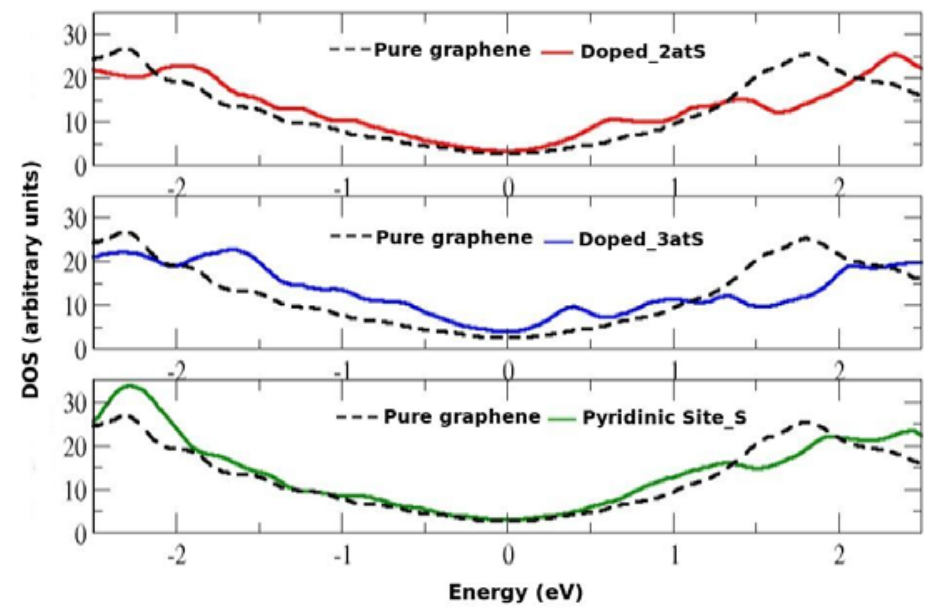

FIGURE 14.

AUTHOR: ALE GARCIA.

ARTICLE: INFLUENCE OF S AND P IN A GRAPHENE SHEET 


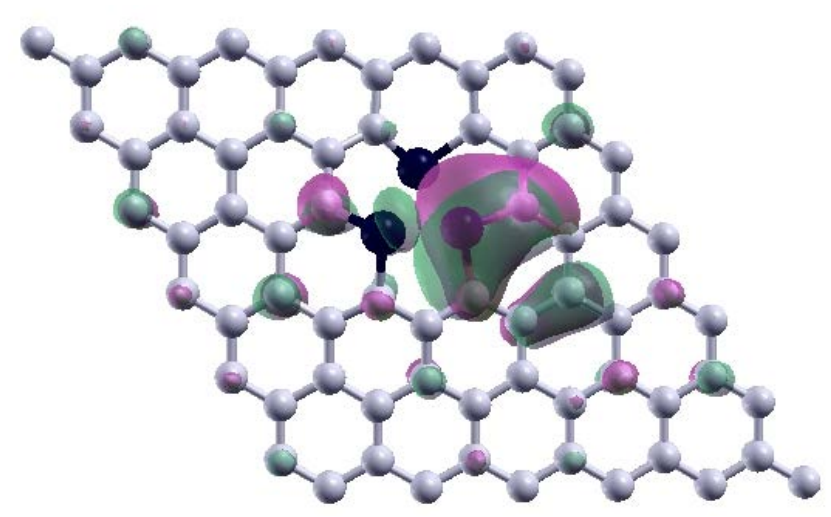

FIGURE 15.

AUTHOR: ALE GARCIA.

ARTICLE: INFLUENCE OF S AND P IN A GRAPHENE SHEET 


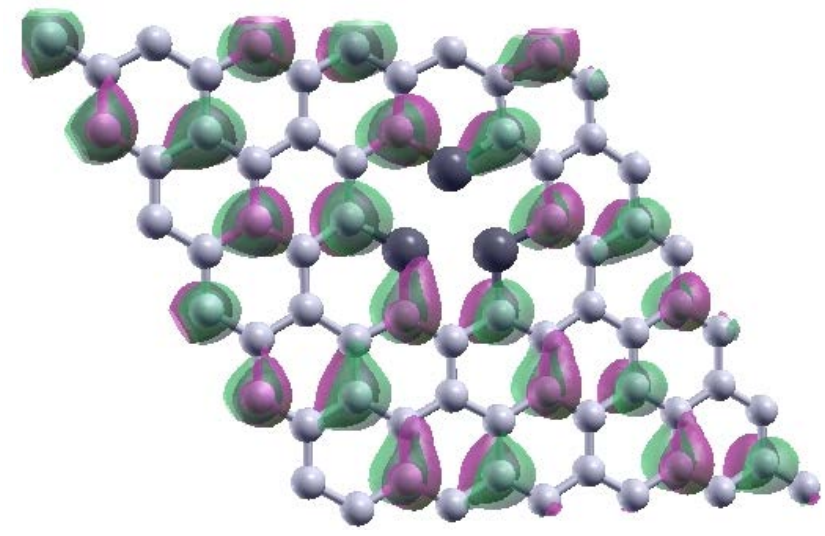

FIGURE 16.

AUTHOR: ALE GARCIA.

ARTICLE: INFLUENCE OF S AND P IN A GRAPHENE SHEET 
TABLE I

\begin{tabular}{cc}
\hline Combination & Formation Energy (eV) \\
AD (sulphur) & 5.98 \\
AC (sulphur) & 11.14 \\
AB (sulphur) & 11.23 \\
AD (Phosphorous) & 0.54 \\
AC (Phosphorous) & 5.53 \\
AB (Phosphorous) & 8.75 \\
\hline
\end{tabular}

AUTHOR: ALE GARCIA

ARTICLE: INFLUENCE OF S AND P IN A GRAPHENE SHEET

TABLE II 


\begin{tabular}{ccc}
\hline Spread & Covalent Bond X-C ( $)$ & Lone pair $(\AA)$ \\
\hline Sulphur (x) & 0.95 & 1.10 \\
\hline Phosphorous (x) & 0.99 & 1.35 \\
\hline
\end{tabular}

AUTHOR: ALE GARCIA

ARTICLE: INFLUENCE OF S AND P IN A GRAPHENE SHEET

TABLE III

\begin{tabular}{cc}
\hline Combination & Energy Formation (eV) \\
\hline Pyridinic site (sulphur) & 9.52 \\
BGF (sulphur) & 10.53 \\
ABC (sulphur) & 22.65 \\
ABD (sulphur) & 28.11 \\
ABE (sulphur) & 28.18
\end{tabular}




\begin{tabular}{cc} 
Pyridinic site (phosphorous) & 0.25 \\
BGF (Phosphorous) & 1.82 \\
ABE (Phosphorous) & 23.31 \\
ABD (Phosphorous) & 27.70 \\
ABC (Phosphorous) & 43.98 \\
\hline
\end{tabular}

AUTHOR: ALE GARCIA

ARTICLE: INFLUENCE OF S AND P IN A GRAPHENE SHEET

TABLE IV

\begin{tabular}{ccccc}
\hline $\begin{array}{c}\text { Formation } \\
\text { Energy }\end{array}$ & $\begin{array}{c}\mathbf{1} \text { dopant atom } \\
(\mathbf{e V})\end{array}$ & $\begin{array}{c}\mathbf{2} \text { dopant } \\
\text { atoms }(\mathbf{e V})\end{array}$ & $\begin{array}{c}\text { 3 dopant atoms } \\
(\mathbf{e V})\end{array}$ & $\begin{array}{c}\text { Pyridinic sites } \\
(\mathbf{e V})\end{array}$ \\
$\begin{array}{c}\text { Sulphur } \\
\text { Phosphorous }\end{array}$ & 5.78 & 7.43 & 10.53 & 9.52 \\
& 2.73 & 0.54 & 1.82 & 0.25 \\
\hline
\end{tabular}

AUTHOR: ALE GARCIA

ARTICLE: INFLUENCE OF S AND P IN A GRAPHENE SHEET 
TABLE V

\begin{tabular}{cccc} 
Spread & $\begin{array}{c}\text { Covalent Bond } \\
\mathrm{A}-\mathrm{C}_{\mathrm{B}}(\AA)\end{array}$ & $\begin{array}{c}\text { Lone pair for } \mathrm{C}_{\mathrm{B}} \text { atom } \\
(\AA)\end{array}$ & $\begin{array}{c}\text { Lone pair for } \\
\text { impurities }(\AA)\end{array}$ \\
Sulphur & 1.05 & 1.12 & 1.08 \\
Phosphorous & 1.01 & 1.38 & 1.35 \\
\hline
\end{tabular}

AUTHOR: ALE GARCIA

ARTICLE: INFLUENCE OF S AND P IN A GRAPHENE SHEET 
TABLE VI

\begin{tabular}{ccc} 
Spread & $\begin{array}{c}\text { Covalent Bond B-C } \\
(\AA)\end{array}$ & $\begin{array}{c}\text { Lone pair for impurities } \\
(\AA)\end{array}$ \\
Pyridinic S & 1.06 & 0.87 \\
Pyridinic P & 1.80 & 0.99 \\
\hline
\end{tabular}

AUTHOR: ALE GARCIA

ARTICLE: INFLUENCE OF S AND P IN A GRAPHENE SHEET 